\title{
Medidas judiciais atinentes à atenção em saúde mental de adolescentes em conflito com a lei
}

\author{
Ricardo Henrique Soares ${ }^{(a)}$ \\ Márcia Aparecida Ferreira de Oliveira ${ }^{(b)}$ \\ Keler Cristina Leite ${ }^{(\mathrm{c})}$ \\ Gustavo Chiesa Gouveia Nascimento ${ }^{\text {(d) }}$
}

Soares RH, Oliveira MAF, Leite KC, Nascimento GCG. Judicial measures related to the mental health ofteenagers in conflict with the law. Interface (Botucatu). 2017; 21(60):123-31.

This research consists of a documental approach performed in the Court for Minors of São Paulo. It includes the judiciary decisions related to the process of forwarding children and teenagers that are users of psychoactive substances for their treatment in the mental health system. This study analyzes the orientation of judicial decisions by their relation to the model of care adopted in real cases. Here, two models are discussed: the psychiatric classic model and the model of care proposed by the psychiatric reform. The results revealed that, in advance, there are not specific orientations that define the health assistance given to children and teenagers. Thus, two conditions for determining the mental health paradigm that is adopted in judiciary decisions are needed: first, an evaluation of the territorial sphere of the patients and second, the knowledge of the specific mental health service that has been indicated for the treatment.

Keywords: Teenagers in conflict with the law. Psychiatric reform. Territoriality.
Com base em uma pesquisa documental realizada na Vara da Infância e Juventude de São Paulo, que continha decisões judiciais determinando o encaminhamento de crianças e adolescentes usuários de substâncias psicoativas para tratamento no campo da saúde mental, este estudo objetivou analisar a orientação das decisões judiciais em relação ao modelo assistencial adotado nos casos concretos: o modelo psiquiátrico clássico ou o modelo de assistência proposto pela reforma psiquiátrica. Os resultados revelaram que, "a priori", não há uma orientação assistencial definida, havendo a necessidade da análise do caso concreto, especialmente em relação a duas condições essenciais para a determinação do paradigma assistencial no campo da saúde mental adotado nas decisões judiciais: avaliação da esfera territorial dos pacientes e o conhecimento do específico serviço de saúde mental indicado para o tratamento.

Palavras-chave: Adolescente em conflito com a lei. Reforma psiquiátrica. Territorialidade.

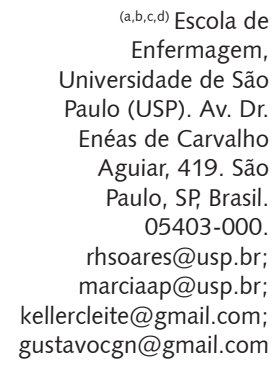

$(a, b, c, d)$ Escola de Enfermagem Universidade de São Paulo (USP). Av. Dr. Enéas de Carvalho Aguiar, 419. São Paulo, SP, Brasil. 05403-000.

rhsoares@usp.br marciaap@usp.br; kellercleite@gmail.com gustavocgn@gmail.com 


\section{Introdução}

Decorrente do princípio da inafastabilidade do controle jurisdicional ${ }^{1}$, a atuação do Poder Judiciário no campo da saúde mental, como em qualquer campo do conhecimento, é fundamental para a manutenção das garantias e dos direitos individuais das pessoas acometidas de sofrimento mental.

Essa atuação específica do Judiciário no campo da saúde mental merece nossa atenção em função do processo de transformação que as teorias e técnicas assistenciais em saúde mental vêm sofrendo nas últimas décadas, com as reivindicações do movimento de Reforma Psiquiátrica em prol da humanização do cuidado e de inclusão de modelos assistenciais alternativos aos tratamentos psiquiátricos asilares, privilegiando modelos baseados em dispositivos de rede comunitária de atenção psicossocial, em detrimento do modelo psiquiátrico tradicional, centralizado no tratamento hospitalar.

Em termos de modelo de atenção em saúde mental, o movimento de Reforma Psiquiátrica privilegia a adoção de tecnologias diversas das de caráter manicomial, propondo um modelo de assistência baseado em dispositivos de rede comunitária de atenção psicossocial, em detrimento do modelo psiquiátrico tradicional, centralizado no tratamento hospitalar.

Comparando as características dos paradigmas da psiquiatria tradicional e da reforma psiquiátrica ${ }^{2,3}$, podemos observar que, enquanto a psiquiatria clássica trata da relação entre o médico e o paciente (doença), a atenção psicossocial de saúde mental trata de uma rede de relações entre sujeitos: cuidadores (assistentes sociais, médicos, enfermeiros, psicólogos, terapeutas ocupacionais, músicos, artistas plásticos, artesãos, etc.) e sujeitos que vivem a experiência do sofrimento mental (usuários e familiares). A psiquiatria tradicional é centralizada, basicamente, no tratamento das doenças, enquanto a atenção psicossocial é centralizada na pessoa - fato que amplia o campo da saúde mental e os serviços constituem dispositivos de trocas sociais. Outra característica fundamental que diferencia os paradigmas da psiquiatria clássica e do serviço de saúde mental e atenção psicossocial se refere ao trabalho de base territorial. A psiquiatria tradicional não desenvolve o serviço em rede territorial, restringindo o serviço à sede da instituição. Para a atenção psicossocial, o serviço de base territorial é imprescindível, e visa desenvolver, ao máximo, as relações entre os usuários e seus familiares com os recursos existentes na comunidade, criando alianças sociais com diversos setores, valendo-se da intersetorialidade como forma de compor uma articulação e cooperação simultânea entre diversos seguimentos.

A Reforma Psiquiátrica tenta oferecer uma proposta de redução de danos e de desvantagens sociais como alternativa ao tratamento baseado no confinamento dos indivíduos acometidos de transtornos mentais e de usuários de substâncias psicoativas4.

No âmbito jurídico, o movimento de Reforma Psiquiátrica se expressa formalmente no Brasil por meio da Política Nacional de Saúde Mental ${ }^{5}$ e pela instituição da lei $10.216 / 2001^{6}$ no ordenamento jurídico vigente. A Política Nacional de Saúde Mental visa a consolidação de um modelo de atenção em saúde mental de base comunitária como substituto do modelo hospitalar especializado, e se apoia na Lei 10.216/2001. Este diploma legal, conhecido como Lei da Reforma Psiquiátrica, caracteriza-se, sobretudo, por assegurar os direitos das pessoas com transtornos mentais sem qualquer forma de discriminação, e reorienta o modelo de assistência em saúde mental para serviços, preferencialmente, extra-hospitalares.

É no cerne do processo de mudanças teórico-práticas no cuidado em saúde mental e no contexto de implantação de políticas públicas frente à condição de pessoas usuárias de substâncias psicoativas que o presente estudo se propôs analisar as decisões do Poder Judiciário no campo da saúde mental, delimitando a problemática de análise dessas decisões ao âmbito de crianças e adolescentes usuários de substâncias psicoativas, especialmente com relação à orientação teórico-técnica do campo da saúde mental adotada pelo Judiciário, com o objetivo de responder a questão: as decisões do Poder Judiciário no campo da saúde mental estão orientadas para o modelo de assistência psiquiátrica tradicional ou para o modelo de assistência proposto pela reforma psiquiátrica? 


\section{Método}

Este é um estudo de abordagem qualitativa, norteado pela questão dos modelos de assistência psiquiátrica e da reforma psiquiátrica no âmbito de decisões judiciais. É baseado numa dissertação de mestrado do Programa de Pós-Graduação da Escola de Enfermagem da Universidade de São Paulo7.

Os dados empíricos foram coletados dos documentais de processos judiciais do acervo de um das Varas Especiais da Infância e da Juventude da Comarca de São Paulo, a partir da concessão da respectiva autorização judicial, bem como pela aprovação no competente Comitê de Ética em Pesquisa da Escola de Enfermagem da Universidade de São Paulo.

A seleção dos processos judiciais do acervo de uma das Varas Especiais da Infância e da Juventude da Comarca de São Paulo se deu no mês de maio de 2012, e seguiu critério não probabilístico, incluindo processos que continham decisões judiciais envolvendo o encaminhamento judicial aos serviços de saúde mental, restando selecionados cinco processos. Os documentos dos processos abrangeram não apenas os documentos referentes às decisões dos juízes, mas os demais documentos que instruíram o processo, como manifestações dos órgãos do Ministério Público, da Defensoria Pública e da Fundação CASA (Centro de Atendimento Socioeducativo ao Adolescente), uma vez que tais documentos estabeleciam uma intertextualização em cada processo.

Com vistas a preservar a confidencialidade e o sigilo das informações contidas nos documentos forenses, que são protegidos legalmente pelo segredo de justiça ${ }^{1,8,9}$, os fragmentos dos textos foram reproduzidos de modo a não permitirem a identificação das partes envolvidas.

\section{Resultados}

\section{Decisões judiciais de encaminhamento aos serviços de saúde mental}

- Primeiro processo: a decisão judicial determinou a medida socioeducativa de Liberdade Assistida, pelo prazo de seis meses, cumulada com medida protetiva do artigo 101, inciso VI, do Estatuto da Criança e do Adolescente (E.C.A.). Nessa decisão, o juiz afirma expressamente que o deferimento da medida protetiva se deve ao requerimento da Defesa do adolescente, no qual há a indicação específica do CAPSad (Centro de Atenção Psicossocial Álcool e Drogas) como aparelho de saúde mental destinado ao tratamento do adolescente.

- Segundo processo: a decisão é composta de medida socioeducativa de Semiliberdade sem prazo determinado e de medida protetiva do artigo 101, inciso VI, do E.C.A., constando expressamente, na instrução processual, que as partes: o Ministério Público e a Defensoria Pública acordaram no sentido de encaminhar o jovem para tratamento no CAPSad.

- Terceiro processo: a decisão homologa a remissão concedida pelo Ministério Público ao adolescente e comina a este medida protetiva do artigo 101, inciso V, do E.C.A. O juiz atribui, em sua decisão, que esta medida protetiva por ele decretada atende ao pedido feito pelo Ministério Público, que, expressamente, requereu o encaminhamento do adolescente para "tratamento psicológico".

- Quarto processo: apresenta duas decisões judiciais relativas ao campo da saúde mental. A primeira delas foi tomada durante a instrução processual, na qual foi determinado o encaminhamento do adolescente ao CAPS-Sé "para avaliar se é necessária internação compulsória para tratamento de drogadição, especialmente no SAID". Nessa decisão, o juiz requer o parecer da equipe técnica do CAPS da região Sé para decidir sobre a eventual internação compulsória do adolescente para o tratamento. Além disso, caso o parecer seja favorável à internação, a decisão indica, expressamente, o respectivo serviço de saúde mental para onde o adolescente deverá ser encaminhado, qual seja, para o SAID (Serviço de Atenção Integral ao Dependente - instituição ligada ao Hospital Samaritano com foco no tratamento de dependentes químicos). Na sentença judicial, apresenta-se a segunda decisão 
de âmbito da saúde mental, consistente na aplicação, ao adolescente, da medida socioeducativa de Internação, sem prazo determinado, cumulada com medida protetiva do artigo 101, VI, do E.C.A. Neste processo, a Fundação CASA se manifesta no sentido de que o jovem "poderá frequentar o CAPSad mais próximo de sua unidade de internação".

- Quinto processo: contém a decisão de aplicar, ao adolescente, o cumprimento da medida socioeducativa de Internação, sem prazo determinado, cumulada com "medida de tratamento para drogadição", do artigo 101, VI, do E.C.A. Nesta decisão, consta que o adolescente, em sua audiência de apresentação, "concorda em tratamento para se livrar das drogas".

\section{Discussão}

\section{Orientação das decisões judiciais em relação à assistência em saúde mental}

A partir do exame das decisões judiciais contidas nos cinco processos coletados, podemos verificar, com exceção de um processo, a incidência de duas medidas judiciais simultâneas e distintas aplicadas sobre o mesmo adolescente: as medidas socioeducativas e as medidas protetivas. As primeiras são referidas à dimensão infracional, ou seja, estão afetas ao adolescente tomado enquanto adolescente em conflito com a lei. As medidas protetivas são concernentes ao adolescente considerado como sujeito de cuidados e de proteção ${ }^{8}$.

Podemos observar que a maioria dos processos analisados apresentou, como dispositivo legal para aplicação das medidas protetivas, o inciso VI do artigo 101 do E.C.A. ${ }^{8}$, enquanto apenas um dos processos empregou o inciso $V$ do mesmo diploma legal. A diferença entre esses dispositivos relaciona-se ao alcance do tratamento em saúde. Enquanto o inciso o VI destina-se especificamente ao tratamento em saúde mental de crianças e adolescentes usuários de substâncias psicoativas, o inciso $\checkmark$ tem uma dimensão ampla, abrangendo a esfera da saúde no âmbito geral da medicina e da saúde mental, seja no campo da psicologia ou no campo da psiquiatria.

Outra consideração pertinente a esses processos está relacionada à menção específica do serviço de saúde mental destinatário dos encaminhamentos. Constatamos que, em três processos, há a indicação expressa do CAPSad (Centro de Atenção Psicossocial Álcool e Drogas) como o respectivo aparelho de saúde mental para onde os adolescentes devem ser encaminhados para tratamento. No primeiro processo, o pedido expresso de encaminhamento ao CAPSad é feito pela Defensoria Pública. Já no segundo processo, esse requerimento é realizado em consenso pelo Ministério Público e pela Defensoria Pública. O quarto processo é referido ao CAPS expressamente pelo próprio juiz, ao requisitar uma avaliação, bem como pela equipe técnica da Fundação CASA.

Os demais processos (terceiro e quinto) não especificam expressamente o serviço de saúde mental para o qual os adolescentes devem ser encaminhados. Todavia, podemos realizar algumas inferências a partir da análise contextual desses processos.

O terceiro processo apresenta a homologação do juiz em face dos requerimentos do Ministério Público: remissão e aplicação de medida protetiva do artigo 101, inciso V, do E.C.A.. Esse dispositivo legal, que garante a possibilidade de o juiz requisitar "tratamento médico, psicológico ou psiquiátrico, em regime hospitalar ou ambulatorial", aparece no requerimento do Ministério Público com a indicação expressa de que a medida protetiva consiste em "tratamento psicológico". Essa ressalva, além de excluir as possibilidades de que o tratamento seja médico ou psiquiátrico, pressupõe que o regime de tratamento não seja hospitalar em virtude do próprio exercício profissional da Psicologia. Ainda podemos inferir que o regime de tratamento não seja hospitalar em razão da manifestação do Promotor de Justiça no sentido de estimar que o uso de drogas feito pelo adolescente se trata de uma questão pontual e circunstancial: "O declarante não é viciado em crack, conhece as consequências nefastas da droga e não mais fará uso de drogas.".

O quinto processo também não indica expressamente o serviço de saúde mental ao qual o jovem é destinado. No entanto, podemos identificar referências alusivas às características dos serviços do CAPS na manifestação técnica da "área psicológica" da Fundação CASA: "Perante o exposto, faz necessário 
o jovem ser estimulado por uma equipe multidisciplinar a desenvolver suas potencialidades e, através dos atendimentos psicossociais, estimulado a valorizar novas possibilidades de vida". Podemos notar, ainda, que, ao aplicar a medida socioeducativa de internação cumulada com medida protetiva, a decisão judicial não determina expressamente que o "tratamento para drogadição" seja feito em regime hospitalar, pressupondo-se que o adolescente permanecerá sob custódia na Fundação CASA, e não em instituições hospitalares.

Observando três processos (primeiro, segundo e quarto) em que há a indicação expressa do CAPS como serviço de saúde mental destinado ao tratamento dos adolescentes, a resposta tenderia a corresponder ao modelo de assistência preconizado pelo movimento de Reforma Psiquiátrica, vez que o CAPS constitui dispositivo central da logística do serviço de saúde mental de rede comunitária, que se propõe como substituto aos serviços de saúde mental centrados no hospital psiquiátrico e na assistência psiquiátrica tradicional. No entanto, se nos detivermos mais atentamente à totalidade da espécie, poderemos constatar que essa conclusão pode ser precipitada em função da aplicação de determinadas medidas socioeducativas em conjugação com o encaminhamento ao CAPS, de modo que devemos examinar a compatibilidade entre o cumprimento das medidas socioeducativas e o tratamento oferecido no CAPS, buscando verificar se este serviço conserva seu potencial e seu sentido dentro da lógica da rede comunitária de saúde mental, para podermos responder à questão de pesquisa.

Analisando, em primeiro lugar, a medida socioeducativa de internação agregada ao encaminhamento ao CAPS (quarto processo), constatamos, desde logo, a incidência de uma contradição entre essa medida privativa de liberdade e a lógica do CAPS como serviço de saúde mental em rede de base comunitária. Isso porque a aplicação da medida de internação interfere diretamente na dimensão do território do adolescente, não somente em decorrência da limitação espacial dada pelo regime de clausura próprio da medida de internação, mas em função do sentido social e simbólico conferido à concepção de território dentro da lógica da atenção psicossocial.

O conceito de território no âmbito da saúde mental em rede comunitária está ligado a uma noção integradora, não identificada somente como espaço natural nem apenas como espaço político, econômico ou cultural, mas considerada numa perspectiva totalizante dessas diferentes dimensões sociais. É nesse território integrado que as pessoas podem construir e exercer, efetivamente, o controle político e econômico e se apropriar dos recursos ${ }^{10}$.

Esse conceito de território é fundamental para a compreensão do serviço em rede de saúde mental, porque a própria rede comunitária pressupõe o território, de modo que falar em rede é, antes, falar em território. Isso significa que é a partir do território que a rede se organiza, propondo-se a substituir o serviço psiquiátrico tradicional (centrado no hospital psiquiátrico) por uma clínica ampliada de atenção psicossocial.

Nessa perspectiva de território relacional, material e simbolicamente integrado, o cumprimento da medida de internação se incompatibiliza com a lógica da rede de atenção comunitária do CAPS, proposta pela Reforma Psiquiátrica, uma vez que a internação (privação da liberdade) do adolescente o impede de se apropriar dos recursos e potenciais do território e de fortalecer seus laços familiares e comunitários, visando à reabilitação psicossocial.

Esse efeito restritivo e limitador imposto à dimensão do território dos adolescentes pela aplicação da medida de internação é denominado, por Haesbaert ${ }^{10}$, de desterritorialização ou territorialização precária. Segundo esse autor ${ }^{10}$, a precarização territorial "deve ser aplicada a fenômenos de efetiva instabilidade ou fragilização territorial, principalmente entre grupos socialmente mais excluídos e/ou profundamente segregados" (p. 312).

O conhecimento a respeito da esfera de territorialidade das crianças e adolescentes encaminhados a tratamento é importante também na medida em que o tipo de assistência oferecida está relacionado à permanência desses usuários no próprio tratamento. Estudo ${ }^{11}$ realizado com o fim de analisar fatores que influenciam a permanência de adolescentes no tratamento no CAPS identificou que a integração do CAPS aos serviços da rede comunitária constitui uma das variáveis essenciais para a permanência desses usuários no serviço; demonstrando que os adolescentes que não se valeram de qualquer tipo de integração entre os diversos dispositivos da rede permaneceram menos tempo no CAPS, em relação 
aos usuários que tiveram acesso aos serviços da rede. Ainda conforme o estudo ${ }^{11}$, a maior permanência do usuário de um CAPS integrado à rede comunitária se deve ao reconhecimento desse usuário de que ele faz parte da comunidade, e essa percepção de pertencimento pode estimulá-lo a permanecer em tratamento.

Nesse sentido, a contradição entre a medida socioeducativa de internação e o tratamento no CAPS estabelece-se a partir do conceito de território, pois, se por um lado, o serviço de saúde mental em rede comunitária demanda a apropriação do território num sentido integrado, de outro lado, o adolescente internado vive num território precário, que o incapacita de se valer dos potenciais do território-rede no processo de reabilitação psicossocial. Essa contradição entre o território precário (do adolescente internado) e o território integrado (do serviço em rede do CAPS) restringe o tratamento de saúde mental à esfera meramente clínica e ambulatorial, realizada no interior do prédio do CAPS, configurando-se numa simples inclusão no CAPS, e não numa inclusão no serviço de rede comunitária e atenção psicossocial proposto pelo CAPS.

Essa mesma contradição poderia recair, também, sobre o quinto processo, que, da mesma forma que o anterior, estabelece medida socioeducativa de internação cumulada com "medida de tratamento para drogadição". E dizemos poderia recair porque não há a indicação expressa do aparelho de saúde mental destinado ao tratamento do jovem, de modo que, se o tratamento envolvesse a atenção psicossocial (e, como mencionado, pressupomos, pelo contexto, que sim), a contradição entre o território integrado da rede de atenção comunitária e a precarização do território do adolescente decorrente da medida de internação também se estabeleceria, constituindo-se como entrave essencial para o tratamento em saúde mental em rede comunitária.

Passando a analisar a conjugação entre a medida de semiliberdade com o encaminhamento ao CAPS (segundo processo), observamos a mesma contradição entre a necessidade de um território integrado para a realização do serviço de saúde mental em rede e o território precário do adolescente em cumprimento de medida de semiliberdade, apesar de o nível de precarização do território do adolescente ser menor do que a desterritorialização provocada pela medida de internação. Ou seja, a despeito de o nível de precarização territorial do adolescente ser inferior, no caso da medida de semiliberdade, já que o adolescente tem o direito de sair durante o dia da instituição socioeducativa para realizar as atividades atinentes a seu programa individual de atendimento, o jovem permanece institucionalizado e desfalcado da territorialidade integrada - circunstância que arrefece o potencial do serviço em rede comunitária e atenção psicossocial do CAPS.

Com relação à cumulação entre medida de liberdade assistida e o tratamento no CAPS (primeiro processo), observamos não haver contradição com relação à territorialidade. Isso porque o regime aberto da medida socioeducativa de liberdade assistida não provoca a precarização do território integrado do adolescente, que está livre para se apropriar das potencialidades e dos recursos do território, assim como o território se apropriar de seus potenciais, numa relação dialética promovida pela rede de atenção psicossocial irradiada e organizada pelo CAPS.

Essa mesma conclusão vale para o terceiro processo, se supusermos que o tratamento em saúde mental determinado pelo juiz deva ser realizado no serviço de estratégia de rede comunitária, pois, neste processo, não há cominação de qualquer medida socioeducativa, apenas a medida protetiva, "consistente em tratamento psicológico", de modo que a noção de territorialidade do adolescente não seria comprometida por medidas socioeducativas. Todavia, neste processo, não há indicação expressa do respectivo serviço de saúde mental destinado ao adolescente (como mencionado anteriormente).

A partir dessa discussão dos processos, que resultou na identificação do território como elemento de contradição estabelecido na relação entre o tratamento em saúde mental e o cumprimento de determinadas medidas socioeducativas, podemos considerar que a resposta à questão sobre a orientação assistencial das decisões judiciais no campo da saúde mental está ligada à apreciação da esfera do território do paciente, condicionada pelo potencial desterritorializante de determinadas medidas socioeducativas, notadamente as medidas de internação e de semiliberdade. Além da análise do potencial do território do paciente, a resposta à questão da respectiva orientação assistencial impõe o conhecimento do dispositivo de saúde mental específico destinado ao tratamento dos adolescentes. 
Com efeito, a resposta à questão sobre a orientação das decisões judiciais em relação à assistência em saúde mental depende da prévia avaliação do território do paciente e do conhecimento do respectivo serviço de saúde mental indicado para seu tratamento. Não há a possibilidade de assegurarmos uma resposta prévia à análise da perspectiva do território do paciente e do dispositivo de saúde mental específico, sob pena de incorrermos no equívoco de classificarmos uma decisão judicial orientada pelos paradigmas da Reforma Psiquiátrica, considerando, por exemplo, que a mera inclusão no CAPS configura a inclusão em serviço de saúde mental em rede de base comunitária do CAPS.

Nesse contexto, podemos responder à questão deste estudo nos casos concretos contidos nos processos que revelam o respectivo serviço de saúde mental de encaminhamento dos adolescentes, restando avaliar a perspectiva do território destes jovens para classificarmos a orientação teóricoassistencial do tratamento.

Como anteriormente mencionado, três dos cinco processos contêm indicações expressas sobre o dispositivo de saúde mental específico para o tratamento dos jovens, condição que exclui os outros dois processos (terceiro e quinto) da possibilidade de respondermos sobre o respectivo paradigma de orientação.

Com referência à territorialidade dos adolescentes dos três processos que especificaram o CAPS como o serviço de saúde mental destinado ao tratamento dos adolescentes, o segundo e terceiro processo aplicam medidas socioeducativas (semiliberdade e internação) que precarizam o território dos adolescentes pela institucionalização, enquanto a medida socioeducativa do primeiro processo (liberdade assistida) preserva a territorialidade do jovem.

Com efeito, excluindo os processos que não contemplam a indicação do serviço de tratamento em saúde mental, podemos afirmar que o primeiro processo encontra abrigo no paradigma da Reforma Psiquiátrica e, os demais, no paradigma da psiquiatria tradicional em função de a inclusão dos adolescentes no CAPS não corresponder à inclusão dos jovens no serviço em rede de base comunitária organizado pelo CAPS, constituindo mera inserção dos pacientes no CAPS.

\section{Processos de psiquiatrização}

Além das considerações feitas a respeito das medidas protetivas e os efeitos potencialmente limitadores das medidas socioeducativas que recaem sobre a esfera da territorialidade dos adolescentes, podemos analisar, também, a combinação dessas medidas sob a ótica dos processos de psiquiatrização, a fim de nos auxiliar a refletir sobre as bases de entendimento da dupla aplicação dessas medidas.

A psiquiatrização envolve a expansão do campo da psiquiatria para outras áreas do conhecimento e outros espaços sociais (educação, justiça, trabalho), circunscrevendo-se em relação a comportamentos, práticas e discursos tidos como anormais ${ }^{12}$.

Os processos de psiquiatrização encobrem a leitura dos fenômenos sociais que determinam a condição de desigualdade econômica, política e cultural dos jovens em conflito com a lei, afastando a compreensão da situação de vulnerabilidade e de exclusão social que também determina a própria prática infracional ${ }^{13}$.

Nesse sentido, a aplicação de medidas protetivas de tratamento em saúde mental, conjugadas com medidas socioeducativas, representaria o entendimento de que o adolescente em conflito com a lei está sujeito, por um lado, ao cumprimento de uma medida de caráter expiatório (medida socioeducativa) pela prática do ato infracional, e, de outro lado, está sujeito a tratamento em saúde mental.

Desse modo, os processos de psiquiatrização, também entendidos como "a predominância dos saberes e fazeres psi na gestão das problematizações e dos conflitos que setores da juventude vêm colocando ao campo social"14 (p. 62), manifestam-se na dupla aplicação das medidas socioeducativas e protetivas. 


\section{Conclusão}

Em face da dupla aplicação de medidas socioeducativas e protetivas de tratamento em saúde mental aos adolescentes em conflito com a lei, identificamos a possibilidade de expressão de processos de psiquiatrização no espaço forense.

A contradição estabelecida entre o tratamento em saúde mental de atenção psicossocial e as medidas socioeducativas de internação e semiliberdade, referente à precarização da territorialidade dos adolescentes, revelou duas condições essenciais para a determinação do respectivo paradigma teóricoassistencial do campo da saúde mental, quais sejam: a avaliação da esfera territorial dos adolescentes e o conhecimento do específico dispositivo de saúde mental indicado para o tratamento dos jovens.

A omissão em analisar simultaneamente essas duas condições pode nos destinar a supor equivocadamente, por exemplo, que o tratamento oferecido pelo CAPS a um adolescente que esteja cumprindo medida socioeducativa de internação ou semiliberdade possa encontrar abrigo no referencial da reforma psiquiátrica, quando, na verdade, este jovem está meramente incluído no serviço clínico-ambulatorial prestado na sede do CAPS, não estando, portanto, inserido no serviço de rede comunitária de atenção psicossocial proposto pela reforma psiquiátrica ao dispositivo CAPS.

Com efeito, identificamos um processo, dentre os analisados, que se configurou pertinente e conforme os preceitos da reforma psiquiátrica, já que satisfez os dois requisitos: nomeou o dispositivo específico de tratamento em saúde mental (CAPS) e aplicou uma medida socioeducativa (liberdade assistida), conjugada à protetiva, que não provocou a precarização territorial do adolescente, compatibilizando-se com o serviço em rede comunitária de atenção psicossocial.

Em face da dupla aplicação de medidas socioeducativas e protetivas de tratamento em saúde mental, aos adolescentes em conflito com a lei, também podemos expressar o predomínio dos processos de psiquiatrização no espaço forense.

\section{Colaboradores}

Ricardo Henrique Soares trabalhou na concepção e projeto, análise e interpretação dos dados e redação do artigo. Márcia Aparecida Ferreira de Oliveira trabalhou na concepção e projeto e revisão crítica relevante do conteúdo intelectual. Keler Cristina Leite e Gustavo Chiesa Gouveia Nascimento atuaram na análise e interpretação dos dados. Todos os autores tiveram participação ativa na discussão dos resultados; aprovação final da versão a ser publicada.

\section{Referências}

1. Constituição (1988). Constituição da República Federativa do Brasil. Brasília (DF): Senado Federal; 1988.

2. Amarante P. Loucos pela vida: a trajetória da reforma psiquiátrica no Brasil. 2a ed. Rio de Janeiro: Fiocruz; 1995.

3. Amarante P. Saúde mental e atenção psicossocial. 2a ed. Rio de Janeiro: Fiocruz; 2007.

4. Pitta AMF. Um balanço da reforma psiquiátrica brasileira: instituições, atores e políticas. Cienc Saude Colet. 2011; 16(12):4579-89. 
5. Ministério da Saúde (BR). Secretaria de Atenção à Saúde. DAPE. Coordenação Geral de Saúde Mental. Reforma psiquiátrica e política de saúde mental no Brasil. Documento apresentado à Conferência Regional de Reforma dos Serviços de Saúde Mental: 15 anos depois de Caracas. Brasília (DF): OPAS; 2005.

6. Lei n. 10216, de 6 de abril de 2001. Dispõe sobre a proteção das pessoas portadoras de transtornos mentais e redireciona o modelo assistencial em saúde mental. Diário Oficial da União. 9 Abr 2001.

7. Soares RH. Decisões judiciais no campo da saúde mental [dissertação]. São Paulo (SP): Escola de Enfermagem da Universidade de São Paulo; 2012.

8. Lei n. 8.069, de 13 de julho de 1990. Dispõe sobre o estatuto da criança e do adolescente e dá outras providências. Diário Oficial da União. 17 Jul 1990.

9. Lei 5.869, de 11 de janeiro de 1973. Institui o código de processo civil. Diário Oficial da União. 17 Jan 1973.

10. Haesbaert R. O mito da desterritorialização: do fim dos territórios à multiterritorialidade. Rio de Janeiro: Bertrand Brasil; 2004.

11. Araujo NB, Marcon SR, Silva NG, Oliveira JRT. Perfil clínico e sociodemográfico de adolescentes que permaceram e não permaneceram no tratamento em um CAPSad de Cuiabá/MT. J Bras Psiquiatr. 2012; 61(4):227-34.

12. Vicentin MCG, Assis DAD, Joia JH. O direito de crianças e adolescentes ao cuidado em saúde mental: tensões entre proteção e tutela no caso do uso de drogas. Diké. 2015; 4(1):21-50.

13. Rosa MD, Vicentin MCG. Os intratáveis: o exílio do adolescente do laço social pelas noções de periculosidade e irrecuperalidade. Rev Psicol Polit. 2010; 10(19):107-24.

14. Vicentin MCG, Gramkow G, Rosa MD. A patologização do jovem autor de ato infracional e a emergência de "novos" manicômios judiciários. Rev Bras Cresc Desenvolv Hum. 2010; 20(1):61-9.

Soares RH, Oliveira MAF, Leite KC, Nascimento GCG. Medidas legales de atención en salud mental para adolescentes en conflicto con la ley. Interface (Botucatu). 2017; 21(60): 123-31.

La presente consiste en una investigación documental realizada en la Vara de Infancia y Juventud de São Paulo, Brasil. La misma está referida a decisiones judiciales que determinan el proceso de orientación de niños y adolescentes usuarios de drogas para su tratamiento en el campo de la salud mental. Este estudio propone analizar la orientación de las sentencias judiciales en lo que hace al modelo adoptado en los casos particulares: el modelo psiquiátrico clásico o el modelo de atención propuesto por la reforma psiquiátrica. Los resultados mostraron que no hay "a priori" una orientación asistencial definida, requeriéndose del análisis de los casos de manera individual. El estudio nota que ello ocurre sobre todo em relación con dos condiciones esenciales para determinar el paradigma el cuidado de la salud mental adoptada en las decisiones judiciales: evaluación del ámbito territorial de los pacientes y conocimiento del servicio de salud mental específico que ha sido indicado para el tratamento.

Palabras clave: Adolescentes en conflicto con la ley. Reforma psiquiátrica. Territorialidad. 
